\title{
Medical management of chronic cholestatic liver diseases
}

\author{
Andrea A Gossard FNP, Keith D Lindor MD
}

\begin{abstract}
AA Gossard, KD Lindor. Medical management of chronic cholestatic liver diseases. Can J Gastroenterol 2000;14(Suppl D): 93D-98D. The purpose of the present review is to discuss the diagnosis and management of cholestatic liver diseases. Differential diagnoses to consider are described, including causes of extrahepatic biliary obstruction such as gallstones, strictures, extrabiliary malignancies and pancreatitis. In addition, diseases that cause intrahepatic cholestasis such as primary biliary cirrhosis, primary sclerosing cholangitis, hepatocellular diseases and a variety of miscellaneous causes including drugs that may cause cholestasis are discussed. Primary biliary cirrhosis and primary sclerosing cholangitis are reviewed in detail, and management options are identified. The prognosis of patients with these diseases is discussed, and the Mayo Mathematical Models in Cholestatic Liver Disease for both primary biliary cirrhosis and primary sclerosing cholangitis are provided. Finally, management options for the complications of cholestasis are provided.
\end{abstract}

Key Words: Cholestasic liver disease; Primary biliary cirrhosis; Primary sclerosing cholangitis

\section{Traitement médical des maladies hépatiques cholestatiques chroniques}

RÉSUMÉ : Le présent article passe en revue le diagnostic et le traitement des maladies hépatiques cholestatiques. Les diagnostics différentiels dont il faut tenir compte sont décrits, y compris les causes extrahépatiques de l'obstruction biliaire, comme les calculs biliaires, les structures, les néoplasies extrabiliaires et la pancréatite. De plus, les maladies qui causent la cholestase intrahépatique, comme la cirrhose biliaire primaire, la cholangite sclérosante primaire et les maladies hépatocellulaires, de même qu'une variété de causes diverses, notamment les toxicomanies qui peuvent provoquer la cholestase sont abordées. La cirrhose biliaire et la cholangite sclérosante primaire sont passées en revue plus en détail, de même que les options thérapeutiques. On passe également en revue le pronostic des patients atteints de ces maladies et l'on fournit quelques modèles mathématiques de Mayo sur les maladies hépatiques cholestatiques pour la cirrhose biliaire primaire et la cholangite sclérosante primaire. En dernier lieu, l'article propose certaines options thérapeutiques pour les complications de la cholestase.
$\mathrm{C}$ hronic cholestatic liver diseases are not common, but they are nonetheless important. The medical management of such diseases depends on identifying as precisely as possible the cause of the chronic cholestasis, the exclusion of reversible conditions and the selection of appropriate therapy when possible for specific entities, as well as the recognition and management of cholestasis-specific complications.

\section{DIAGNOSIS}

Symptoms: Symptoms are usually nonspecific. Many patients with chronic cholestatic liver diseases are brought to attention only because of mild abnormalities found in liver tests, particularly in the alkaline phosphatase level, on routine testing. Some patients may come to attention because of hypercholesterolemia. Occasionally, pruritus, fever and right upper quadrant pain are present.

Biochemistry: Biochemical changes are relatively nonspecific. The alkaline phosphatase level is prominently elevated. Gamma glutamyl transpeptidase is frequently elevated but is a nonspecific abnormality. The aminotransferase concentrations are usually modestly elevated. Hyperbilirubinemia is unusual at presentation of chronic cholestatic liver diseases but may become markedly elevated late in the course. Serum cholesterol levels may be elevated. Various autoantibodies such as the antimitochondrial antibody may be present.

This mini-review was prepared from a presentation made at the American Congress of Gastroenterology, Boston, Massachusetts,

October 9 to 11,1998

Division of Gastroenterology and Hepatology, Mayo Clinic and Foundation, Rochester, Minnesota

Correspondence and reprints: Dr Keith D Lindor, Division of Gastroenterology and Hepatology, Mayo Clinic, 200 First Street South West,

Rochester, Minnesota 55905, USA. Telephone 507-284-1738, fax 507-266-4531, e-mail lindor.keith@mayo.edu

Received for publication June 7, 1999. Accepted June 13, 1999 
TABLE 1

Extrahepatic causes of biliary obstruction

\begin{tabular}{l} 
Intraductal obstruction/abnormalities \\
Gallstones \\
Strictures \\
Malignancy of bile ducts \\
Malformation \\
Autoimmune disease cholangiopathy \\
Extrinsic compression \\
Extrabiliary malignancies \\
Pancreatitis \\
Lymphoma \\
\hline
\end{tabular}

TABLE 2

Intrahepatic causes of cholestasis

\begin{tabular}{l} 
Primary biliary cirrhosis \\
Primary sclerosing cholangitis \\
Hepatocellular disease \\
Alcoholic hepatitis \\
Hepatitis B \\
Hepatitis C \\
Autoimmune hepatitis \\
Miscellaneous causes \\
Sarcoidosis \\
Idiopathic adult ductopenia \\
Benign recurrent intrahepatic cholestasis \\
Cystic fibrosis \\
Drug-induced cholestasis \\
\hline
\end{tabular}

Imaging: Cross-sectional imaging with ultrasound or computed tomography scanning in some instances, depending on the situation and body habitus, are useful for excluding biliary obstruction as a cause of cholestasis. When suspicion is high or signs on cross-sectional imaging point to mechanical obstruction, direct cholangiography either endoscopically or occasionally percutaneously is necessary. Cholangiography is necessary to diagnose primary sclerosing cholangitis (PSC). However, cholangiography is seldom needed in the usual clinical setting of chronic cholestasis in a middle-aged woman, with positive antimitochondrial antibodies and negative cross-sectional imaging studies, in whom primary biliary cirrhosis (PBC) is expected.

Many entities in the differential diagnosis may be excluded by the patient's history, and basic laboratory and imaging studies. A liver biopsy is useful in many instances to define better the cause of cholestasis, particularly when suspecting unusual conditions such as autoimmune cholangitis, overlap syndromes and patients with suspected sarcoidosis.

\section{DIFFERENTIAL DIAGNOSIS}

The differential diagnosis must consider extrahepatic causes of biliary obstruction and diseases that cause intrahepatic cholestasis (Tables 1,2). Drug toxicity must always be considered. Common drugs that may cause cholestasis and their patterns of injury are listed in Table 3.

\section{TABLE 3}

Common drugs that may cause cholestasis

\begin{tabular}{l} 
Acute cholestatic hepatitis \\
Azathioprine \\
Captopril \\
Chloropromazine \\
Chlordiazepoxide \\
Cyclosporine \\
Erythromycin \\
Gold salts \\
Acute cholestasis (bland) \\
Oral contraceptives and estrogen \\
Anabolic steroids and androgens \\
Granulomatous hepatitis \\
Allopurinol \\
Carbamazepine \\
Hydralazine \\
Penicillin \\
Quinidine \\
Sulphonamides \\
Mixed hepatitis \\
Amitriptyline \\
Benoxoprofen \\
Carbamazepine \\
Cimetidine \\
Dapsone \\
Imipramine \\
Quinidine \\
Ranitidine \\
Sulphonamides \\
Sulindac \\
Chronic cholestasis \\
Ajorouracil or floxuridine \\
\hline
\end{tabular}

\section{CAUSES OF CHOLESTATIC LIVER DISEASE}

Extrahepatic causes of chronic cholestatic liver disease should be excluded early because they are potentially reversible; failure to do so may result in complications such as recurrent cholangitis or secondary biliary cirrhosis.

Hepatocellular diseases that may cause intrahepatic cholestatic liver disease include viral hepatitis B and C. Hepatitis $\mathrm{C}$ has been associated with chronic cholestasis, although the usual biochemical abnormalities reflect hepatocellular damage. These patients have antibodies to the hepatitis $\mathrm{C}$ virus and are antimitochondrial antibody negative. Alcoholic hepatitis may present with clinical and biochemical features of cholestatic liver disease. This type of hepatocellular disease may also coexist with other liver diseases; therefore, a detailed history is essential.

Autoimmune hepatitis may present quite similarly to $\mathrm{PBC}$, especially in patients with autoimmune hepatitis who are also positive for antimitochondrial antibodies. However, 


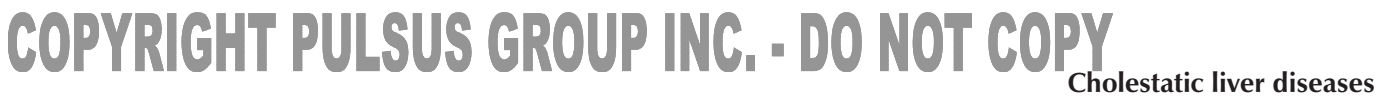

the majority of patients with autoimmune hepatitis have antinuclear antibodies and antismooth muscle antibodies in the serum; on liver biopsy these patients rarely have destruction of the bile ducts. Pruritus is less common, and the serum alkaline phosphatase level is minimally elevated. In $2 \%$ to $3 \%$ of cases, it may be essentially impossible to differentiate between autoimmune hepatitis and PBC, but under these circumstances patients with autoimmune hepatitis experience a dramatic response to a trial of immunosuppressive therapy.

Chronic cholestatic sarcoidosis, a disease most common in young black men, can present with features that are histologically similar to PBC and in some cases PSC. Granulomatous destruction of small ducts is a hallmark of this condition. The granulomas are typically large and well defined, in contrast to the small and poorly defined granulomas seen in PBC. Most of these patients have hilar adenopathy on chest roentgenograms and other features of sarcoidosis, but do not have antimitochondrial antibodies.

Idiopathic adulthood ductopenia is an unusual syndrome that occurs in individuals who have normal cholangiograms, negative antimitochondrial tests and no associated inflammatory bowel disease. These patients present with chronic cholestasis and have ductopenia on liver biopsy. The natural history of this disease is unknown.

Benign familial recurrent cholestasis is a rare syndrome characterized by recurrent attacks of pruritus and jaundice. During the attack, serum alkaline phosphatase levels rise and the liver biopsy reveals cholestasis. The cholangiogram is normal, however, and all cases eventually go into remission. Cirrhosis does not develop, and the prognosis is excellent.

Patients with cystic fibrosis may have neonatal jaundice that generally resolves but recurs later in life. Histological examination of the liver reveals mucus-plugged cholangioles without significant parenchymal damage; consequently portal hypertension is a prominent manifestation, whereas liver failure is rare. Approximately one-third of patients have abnormalities of the gallbladder or cystic duct. Prognosis is largely determined by the status of the pulmonary disease.

The two most common causes of chronic intrahepatic cholestatic liver disease in adults are PBC and PSC $(1,2)$. $\mathrm{PBC}$ and $\mathrm{PSC}$ progress slowly and are important indicators for liver transplantation. These two diseases have a number of features in common but also have important differences. Effective treatment is available for PBC; however, no effective therapy has been identified for patients with PSC. Survival models may be helpful in predicting the prognosis of these two diseases. Each of these diseases has a number of complicating conditions that they may be associated with. It is important to recognize the complicating conditions because specific therapies are available for some of them.

\section{NATURAL HISTORY}

$\mathrm{PBC}$ can have a very long natural history (3). Earlier, it was thought that the median survival after diagnosis was approximately 10 years, but this was primarily due to patients presenting with more advanced disease. Asymptomatic patients may have a slightly longer course, but over time, generally develop symptoms and follow a course very similar to that of patients who present with symptoms earlier in the course of the disease. The natural history of PSC is not as well defined, although this disease also runs a progressive course. Patients may be asymptomatic, but as the disease progresses, symptoms often develop. Patients with PSC may have an illness that lasts between 10 and 20 years, depending on the stage of disease at presentation.

Histological staging can be used to help provide prognostic information, but concerns about sampling variability in PBC and PSC are problematic. Survival models that can help to assess prognosis have been developed independent of histological staging.

These diseases are relatively uncommon. It is estimated that in the United States the prevalence of PBC is about 150 cases/million people, although recent natural history information from a stable population in England suggests a dramatic increase in PBC that is unexplained (4). The prevalence of PSC is estimated to range from 50 to 70 cases/million people. The diagnosis appears to be made more commonly, but this is probably due to greater clinical awareness and greater accessibility of appropriate diagnostic testing.

\section{DIAGNOSTIC FEATURES}

$\mathrm{PBC}$ and PSC both present with chronic cholestasis. $\mathrm{PBC}$ is characterized by the presence of antimitochondrial antibodies in over $95 \%$ of patients; however, these titres are not correlated with disease severity (5). Ninety per cent or more of patients with $\mathrm{PBC}$ are women, although men with $\mathrm{PBC}$ follow a similar course. Recently, the term autoimmune cholangitis was used for patients who have clinical and histological features of PBC but who are antimitochondrial antibody negative. These patients are generally positive for antinuclear or antismooth muscle antibodies. Autoimmune cholangitis and PBC appear to be part of a disease spectrum with very similar clinical and histological features, and similar responses to therapy. Liver biopsy is helpful in confirming this disease, but in patients who have prominent cholestatic liver biochemistries and high antimitochondrial antibody levels, the diagnosis of $\mathrm{PBC}$ is highly likely and there is debate as to whether liver biopsy is necessary. Because therapy for this disease is likely to be life-long, a liver biopsy to confirm the diagnosis before starting therapy seems wise. Cross-sectional imaging studies are usually sufficient to exclude obstruction. Endoscopic retrograde cholangiopancreatography (ERCP) is seldom necessary, unless unusual features suggest large duct obstruction or the patient has coexistent inflammatory bowel disease, and PSC is considered.

PSC is strongly associated with inflammatory bowel disease. Approximately $70 \%$ of patients have colitis. Typically chronic ulcerative colitis (CUC) underlies PSC, but occasionally a patient may have Crohn's colitis. Patients with PSC who have inflammatory bowel disease follow a course similar to that of those who do not have inflammatory bowel 
TABLE 4

\section{Histological staging in primary biliary cirrhosis}

\begin{tabular}{|c|c|}
\hline Stage 1 (portal stage) & $\begin{array}{l}\text { Inflammation limited to portal triads with } \\
\text { mononuclear infiltration }\end{array}$ \\
\hline Stage 2 (periportal stage) & $\begin{array}{l}\text { Inflammation beyond portal triads, } \\
\text { piecemeal necrosis, small granulomas, } \\
\text { and small duct proliferation may be seen }\end{array}$ \\
\hline Stage 3 (septal stage) & Bridging fibrosis connecting the portal tracts \\
\hline Stage 4 (cirrhosis) & Regenerative nodules surrounded by fibrosis \\
\hline
\end{tabular}

TABLE 5

Histological staging in primary sclerosing cholangitis

\begin{tabular}{ll}
\hline Stage 1 (portal stage) & $\begin{array}{c}\text { Portal hepatitis (limited to limiting } \\
\text { plate) }\end{array}$ \\
Stage 2 (periportal stage) & $\begin{array}{c}\text { Periportal fibrosis/inflammation, } \\
\text { beyond limiting plate }\end{array}$ \\
Stage 3 (septal stage) & $\begin{array}{l}\text { Septal fibrosis/bridging necrosis } \\
\text { Stage } 4 \text { (cirrhosis) }\end{array}$ \\
\end{tabular}

disease. The only difference appears to be the suggestion that patients with both PSC and CUC are at an increased risk of developing colorectal cancers as well as cholangiocarcinomas. Antimitochondrial antibodies are typically absent in patients who have PSC. Other antibodies, such as antinuclear antibodies and antineutrophil cytoplasmic antibodies are frequently found. Cross-sectional imaging studies are of limited value for patients with suspected PSC. A cholangiogram usually obtained endoscopically is the most important diagnostic test. Magnetic resonance cholangiography is being evaluated and appears to be promising. It is a noninvasive test that does not require administration of contrast or use of radiation. Liver biopsy is useful in histological confirmation and staging of the disease, but sampling variability can be troublesome. Liver biopsies in both PBC and PSC have been done on stages 1 to 4 depending on the degree of inflammation and fibrosis (Tables 4,5). Stage 4 in both diseases is cirrhosis.

Typical cholangiographic features of PSC include multifocal stricturing, usually involving the extrahepatic ducts. Some patients, perhaps up to $20 \%$, may have only intrahepatic duct involvement. Multifocal stricturing and sacular segmental dilation are typical. Occasionally, patients present with histological features of PSC in the setting of chronic colitis but have normal cholangiograms. These patients are considered to have small duct PSC. It is uncertain whether this condition develops into cholangiographically evident PSC, although this has been suggested.

\section{MANAGEMENT OPTIONS}

PBC: A variety of treatment options have been tested in patients with $\mathrm{PBC}$, especially immunomodulatory agents. Therapies that have been tested and been found not to be beneficial nor to have excessive toxicity include penicil- lamine, cyclosporine, azathioprine, thalidomide, malotilate and chlorambucil. Corticosteroids and colchicine, as well as methotrexate have been advocated, although data supporting the use of any of these is either quite limited or unconvincing. The drug with which there is the most experience and optimism is ursodeoxycholic acid. This drug has recently been approved by the United States Food and Drug Administration for use in patients with PBC. Ursodeoxycholic acid is a safe, well-tolerated drug that improves liver biochemistry, and survival free of transplantation and pruritus, and reduces the risk of developing cirrhosis and varices. This is a cost effective therapy and is expected to have a significant impact on the natural history of patients with PBC (6).

Drugs in combination have also been tested. Ursodeoxycholic acid and colchicine have been tested in several studies of patients with PBC, but there is very little evidence that using colchicine with ursodeoxycholic acid adds a benefit compared with ursodeoxycholic acid alone. Methotrexate has been tested in some pilot studies and its benefit seems limited. A large-scale, multicentre, randomized trial is currently ongoing and results will be available within a few years. Until that time, given the toxicity that has been experienced with the combination of methotrexate and ursodeoxycholic acid, empirical methotrexate therapy is not advocated. Corticosteroids, as single agents, have offered some benefit, both biochemically and histologically. A number of studies are now underway in the United States and in Europe to test various corticosteroids in combination with ursodeoxycholic acid.

PSC: Unfortunately, there is no evidence of an effective therapy for PSC. Penicillamine and colchicine have been shown to be ineffective in controlled trials. Ursodeoxycholic acid has been tested and has had limited benefit. In the largest study, biochemistries were improved in patients taking ursodeoxycholic acid, but the length of time until treatment failure was not altered (7). Considerably smaller studies have suggested improvement. Further studies evaluating the use of ursodeoxycholic acid in PSC are underway, and some studies are using higher doses.

A combination of ursodeoxycholic acid and methotrexate was found to be ineffective for patients with PSC in a pilot study. Other pilot studies have explored novel forms of therapy such as pentoxifylline and nicotine, and found these to be ineffective as well. There is no therapy that is advocated for use in patients with PSC.

\section{PROGNOSIS}

Prognosis for both these diseases has been described by mathematical models (Figures 1,2). For PBC, the models rely on age, bilirubin, albumin, prothrombin time and an edema score (8). This model remains useful with ursodeoxycholic acid treatment (9). For patients with PSC, age, bilirubin, presence of varices and aspartate aminotransferase concentration determine the risk score. These models have allowed greater prediction of individual prognosis and have been especially useful in helping in decisions regarding timing of liver transplantation. 


\section{COMPLICATIONS}

Complications of these diseases include those seen in conjunction with advancing liver disease. In addition, patients with cholestasis are predisposed to osteoporosis, pruritus, hypercholesterolemia and fat-soluble vitamin deficiencies. Patients may also experience malabsorption for a variety of reasons including celiac sprue and pancreatic insufficiency.

Complications specific to PSC include the risk of cholangiocarcinoma (incidence of $0.5 \% /$ year), formation of biliary stones, recurrent cholangitis and stomal varices in patients who have undergone a colectomy and ileostomy. Patients with $\mathrm{PBC}$ appear to be at an increased risk of developing hepatocellular carcinoma and may be troubled by the development of keratoconjunctivitis sicca, Raynaud's phenomenon or thyroid insufficiency.

\section{THERAPY FOR THE COMPLICATIONS OF CHOLESTASIS}

Pruritus is a frequent complication of cholestasis. The cause remains unknown but may be accumulation of hydrophobic bile acids or endogenous opioids. This symptom is difficult to manage, and intractable pruritus has occasionally been an indicator for liver transplantation. Cholestyramine, opiate antagonists, ursodeoxycholic acid, rifampin and flumecinol have been used with varying success.

In patients with PBC in North America, osteopenia is nearly always due to osteoporosis. These patients appear to have an accelerated rate of bone loss with age. Most studies suggest that osteopenia is due to decreased formation rather than to increased resorption of bone. Estrogen therapy, especially when instituted soon after menopause, has been associated with slowing of the rate of bone loss. High dose estrogen therapy has a theoretical risk of worsening cholestasis, but this does not appear to be the case with low dose therapy. Nevertheless, it is prudent to reassess the patient clinically and biochemically within two to three weeks after initiation of the estrogen therapy.

Most patients with $\mathrm{PBC}$ and fat-soluble vitamin deficiencies have advanced liver disease, are jaundiced and have a fecal fat loss in the range of 10 to $30 \mathrm{~g} /$ day. Vitamin D deficiency can be confirmed by measuring serum vitamin $D$ levels and when encountered, 50,000 U of water-soluble vitamin D taken once or twice/week is usually sufficient to correct the deficiency. Vitamin A deficiency is uncommon and may present clinically with night blindness. Vitamin A levels can be measured, and when low, 25,000 to 50,000 U two to three times/week should be instituted, starting at the lower levels. The adequacy of replacement therapy should be assessed by repeat serum assays because excessive vitamin A uptake has been associated with hepatotoxicity. Vitamin E deficiency has been reported in a few cases of PBC. Typically, vitamin E deficiency causes a neurological abnormality primarily affecting the posterior columns and characterized by areflexia or loss of proprioception and ataxia. Replacement of vitamin $\mathrm{E}$ in these patients has been disappointing; nevertheless, patients with low levels of vitamin E should be started on replacement therapy, usually $100 \mathrm{mg}$ twice daily.

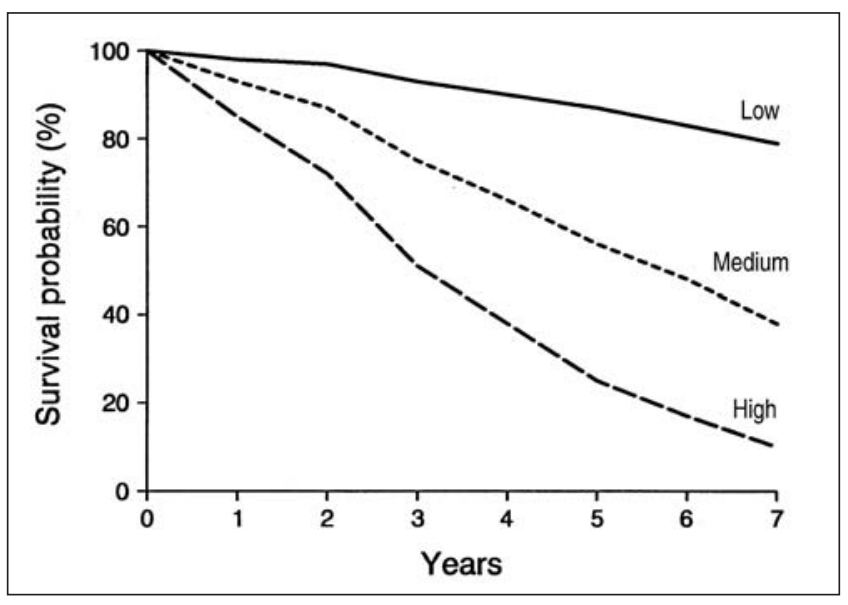

Figure 1) Survival model for primary biliary cirrhosis showing low, medium and high risk patient groups

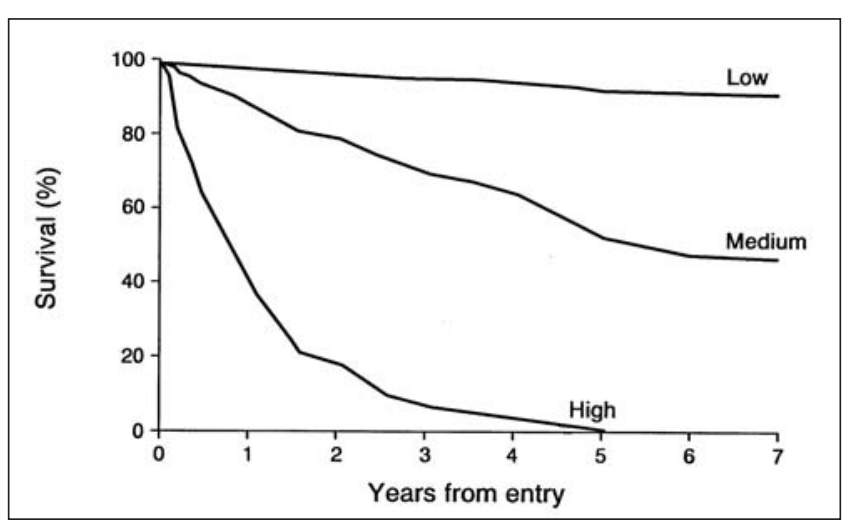

Figure 2) Survival model for primary sclerosing cholangitis showing survival probability for low, medium and high risk patient groups

Hypercholesterolemia is common, especially early in the course of the disease. The incidence of atherosclerotic heart disease is not increased in these patients, and therapy with lipid-lowering drugs is not usually indicated. For patients with cholesterol deposits in the form of xanthomas or xanthelasma, therapy with ursodeoxycholic acid or cholestyramine may stabilize and even decrease the size of these cutaneous deposits.

Malabsorption of nutrients can occur in cholestasis, primarily due to insufficient delivery of bile salts to the intestinal lumen. Celiac sprue has been reported in patients with $\mathrm{PBC}$ and PSC. Furthermore, because of the association of $\mathrm{PBC}$ and scleroderma, wide-mouth diverticula and bacterial overgrowth seen in scleroderma can complicate PBC. In addition, both PBC and PSC have been associated with pancreatic insufficiency, and this must be considered when dealing with patients who have either of these two disorders and evidence of malabsorption.

\section{CONCLUSIONS}

$\mathrm{PBC}$ and PSC are important causes of chronic cholestasis. Effective therapy is now available for patients with $\mathrm{PBC}$, whereas none exists for patients with PSC. Awareness of the 
complications that may be associated with these conditions is helpful when managing these patients. Liver transplantation is an excellent option for patients with clinically advanced disease.

\section{REFERENCES}

1. Kaplan MM. Primary biliary cirrhosis. N Engl J Med 1996;335:1570-80.

2. Harnois DM, Lindor KD. Primary sclerosing cholangitis. Dig Dis 1997;15:23-41.

3. Metcalf JV, Mitchison HC, Plamer JM, Jones DE, Bassendine MF, James OFW. Natural history of primary biliary cirrhosis. Lancet 1996;348:1399-402.

4. Metcalf JV, Bhopal RS, Gray J, Howell D, James OFW. Incidence and prevalence of primary biliary cirrhosis in the city of Newcastle upon Tyne, England. Int J Epidemiol 1997;26:830-6.
5. Kim WR, Poterucha JJ, Jorgensen RA, et al. Does antimitochondiral antibody status affect response to treatment in patients with primary biliary cirrhosis? Outcomes of ursodeoxycholic acid therapy and liver transplantation. Hepatology 1997;26:22-6.

6. Poupon RE, Lindor KD, Cauch-Dudek K, Dickson ER, Poupon R, Heathcote EJ. Combined analysis of French, American, and Canadian randomized controlled trials of ursodeoxycholic acid in primary biliary cirrhosis. Gastroenterology 1997;113:884-90.

7. Lindor KD, and the Mayo PSC/UDCA Study Group. Ursodiol for primary sclerosing cholangitis. N Engl J Med 1997;336:691-5.

8. Grambsch PM, Dickson ER, Kaplan M, LeSage G, Fleming TR, Langworthy AL. Extramural cross-validation of the Mayo Primary Biliary Cirrhosis Survival Model establishes its generalizability. Hepatology 1989;10:846-50.

9. Kilmurry MR, Heathcote EJ, Cauch-Dudek K, et al. Is the Mayo Model for Predicting Survival useful after the introduction of ursodeoxycholic acid treatment for primary biliary cirrhosis? Hepatology 1996;23:1148-53. 


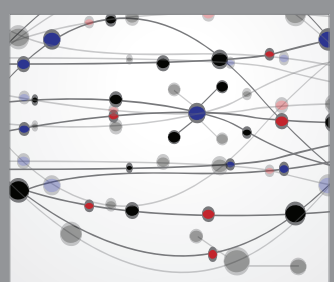

The Scientific World Journal
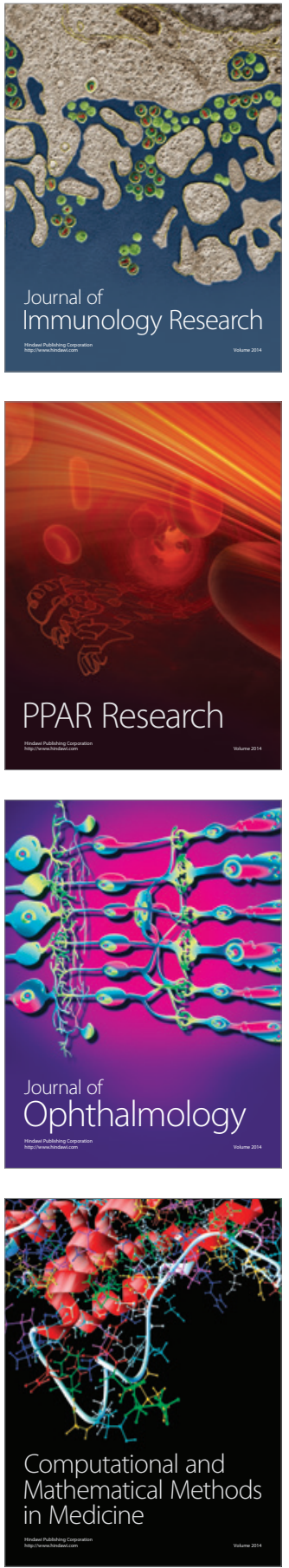

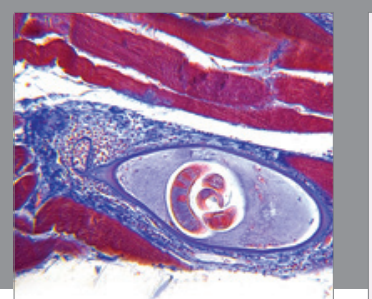

Gastroenterology Research and Practice

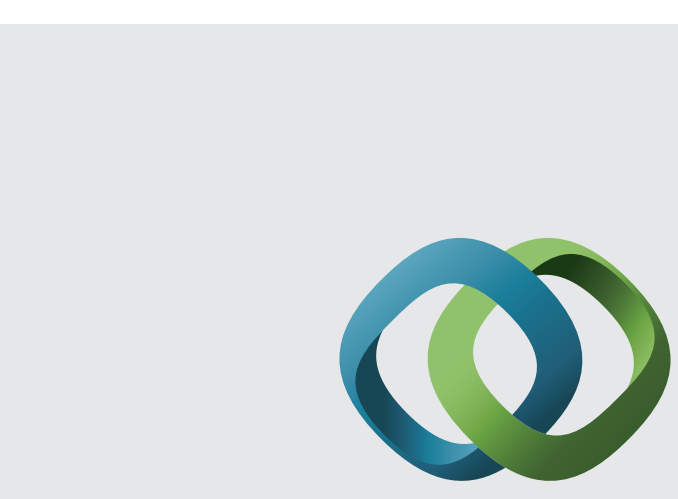

\section{Hindawi}

Submit your manuscripts at

http://www.hindawi.com
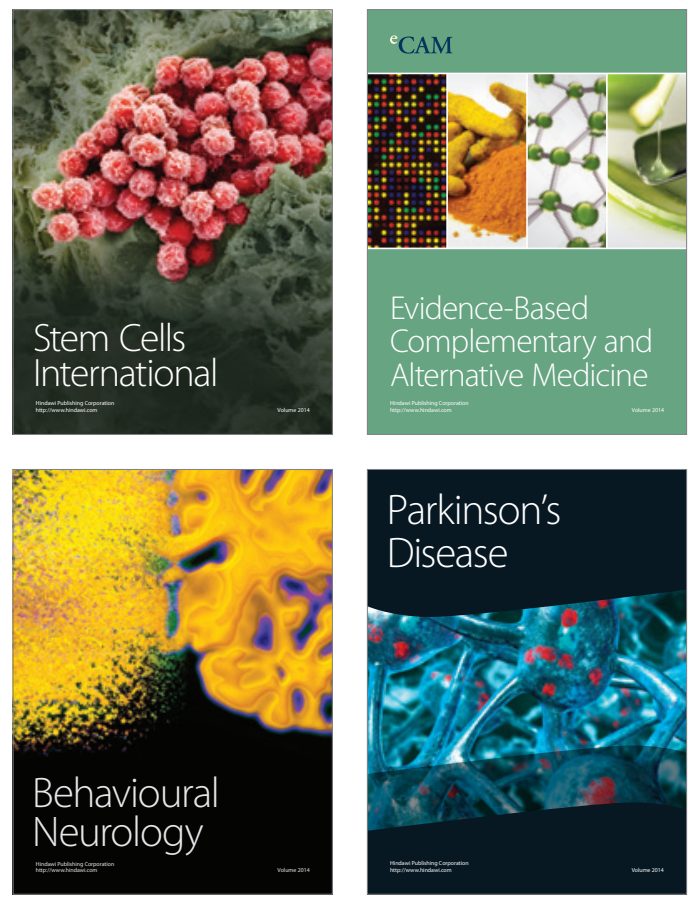
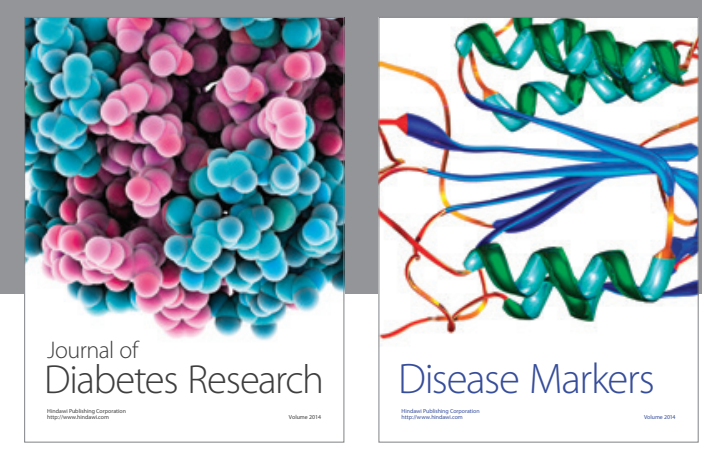

Disease Markers
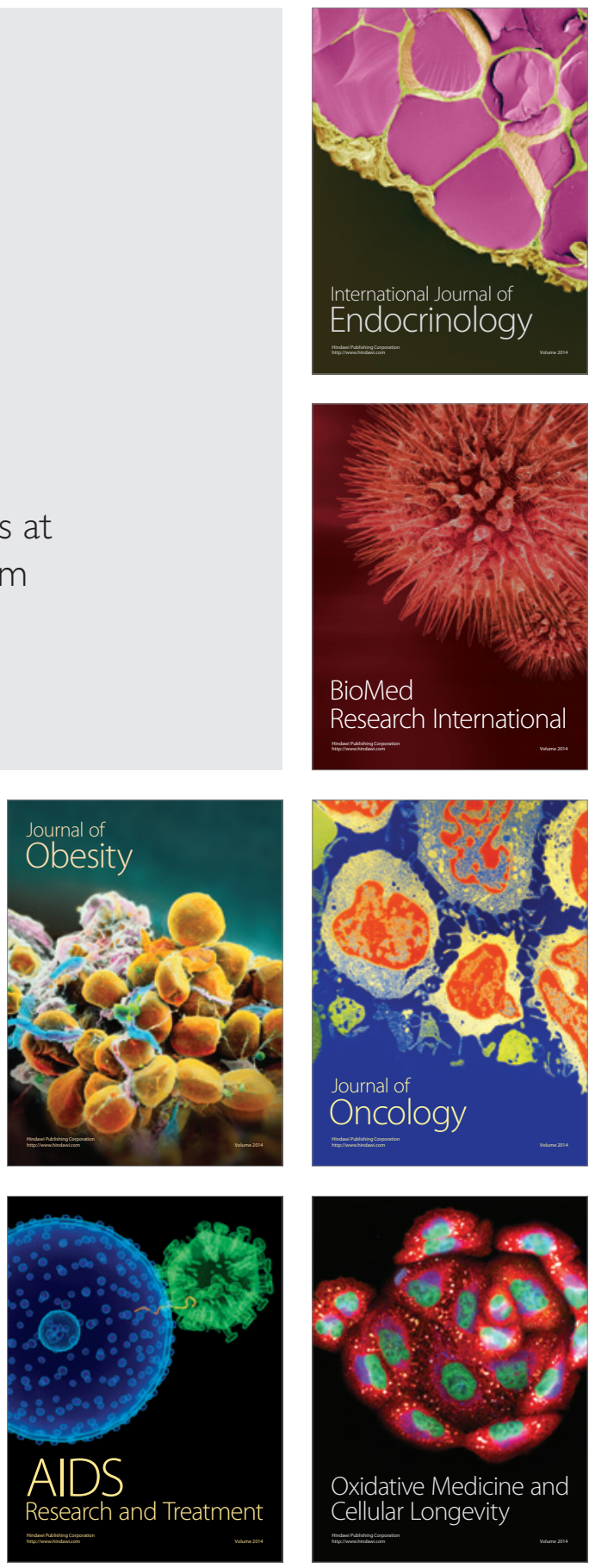